\title{
Sublattice Short-Range Order and Modified Electronic Structure in Defective Half-Heusler $\mathrm{Nb}_{0.8} \mathrm{CoSb}$
}

Shihua Tan ${ }^{1}$, Pengfei Nan ${ }^{2}$, Kaiyang $\mathrm{Xia}^{3}$, Hongliang Yang ${ }^{1,4}$, Tiejun $\mathrm{Zhu}^{3}$, Binghui Ge ${ }^{2, *}$, Wenqing Zhang ${ }^{1,5, *}$

${ }^{1}$ Department of Physics and Shenzhen Institute for Quantum Science \& engineering, Southern University of Science and Technology, Shenzhen, Guangdong 518055, China

${ }^{2}$ Key Laboratory of Structure and Functional Regulation of Hybrid Materials of Ministry of Education, Institutes of Physical Science and Information Technology, Anhui University, Hefei 230601, China

${ }^{3}$ State Key Laboratory of Silicon Materials, School of Materials Science and Engineering, Zhejiang University, Hangzhou 310027, China

${ }^{4}$ Shanghai Institute of Ceramics, Chinese Academy of Sciences, Shanghai, China

${ }^{5}$ Guangdong Provincial Key Lab for Computational Science and Materials Design, and Shenzhen Municipal KeyLab for Advanced Quantum Materials and Devices, Southern University of Science and Technology, Shenzhen, Guangdong 518055, China

*Email: zhangwq@sustech.edu.cn,bhge@ahu.edu.cn
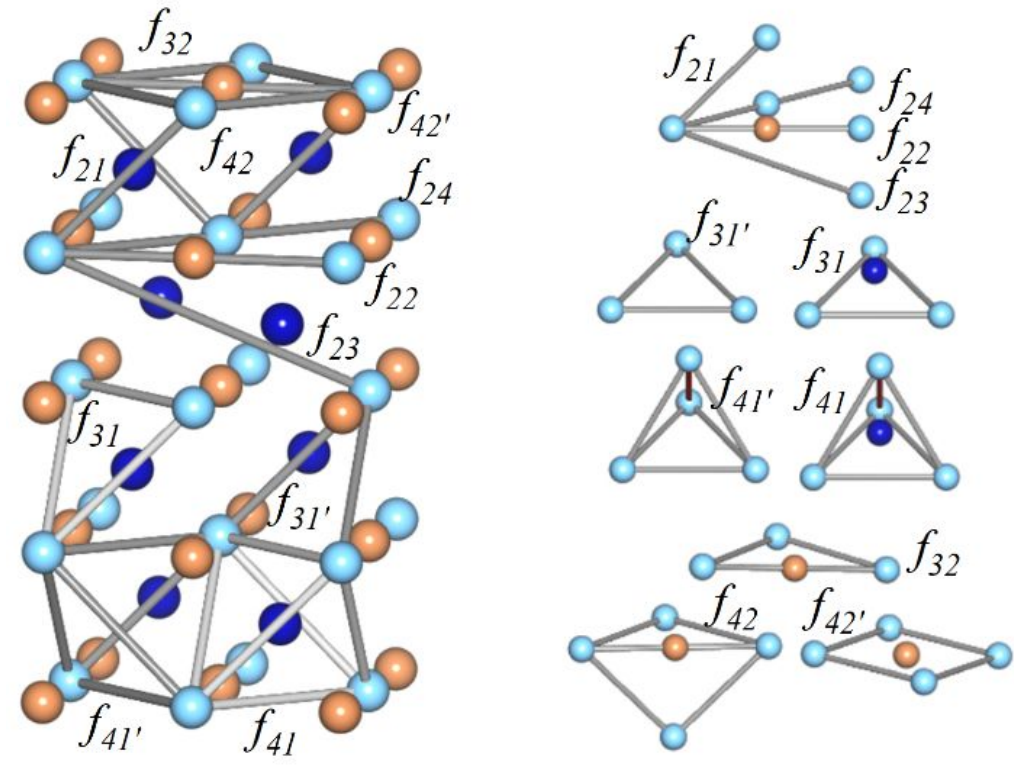

Figure.S1 The 11 selected effective clusters.

\section{Theoretical Methodology}

\section{The Cluster Expansion Method}

The measurable total energy $\bar{E}$ of ordered systems can be obtained by first-principles 
calculations with a small number of atoms in the cell. While the direct first-principles calculations are still impractical in disordered systems which need large cell sizes and thus large number of atoms (e.g., $>10^{3}$ atoms), One of the tools for the study of the properties of systems with disorder characteristics is the cluster expansion (CE) method ${ }^{1-3}$. In a binary $\mathrm{A}_{1-\mathrm{x}} \mathrm{B}_{\mathrm{x}}$ alloy with a lattice of $\mathrm{N}$ sites, there are $C_{N}^{N x}$ arrangements of $N(1-x) \mathrm{A}$ atoms and $N x \mathrm{~B}$ atoms. The statistical lattice theories ${ }^{4,5}$ suggest that, the excess energy $\Delta E(\sigma, V, x)$ of any arrangement (denoted as configuration $\sigma$ ) at constant volume $(V)$ and composition $(x)$, can be discretized into the sum of the elemental properties of constituent component "clusters" $\left\{f_{k m}\right\}$

$$
\Delta E(\sigma, V, x)=E(\sigma, V, x)-E^{0}(V, x)=\sum_{k, m} D_{k m} \bar{\Pi}_{k m}(\sigma) \varepsilon_{k m}(V, x)
$$

Here the $E^{0}(V, x)$ is a reference energy. $k$ represents the number of vertices in one type of cluster, and $k=1,2,3$ corresponds to sites, pairs, and triplets separately. $m$ represents the maximum neighbor distance in the cluster, e.g., $m=1,2$ indicating the first and second neighbors accordingly. Using the language of Ising model, the corresponding spin variable $S_{i}$ is set to be -1 if the site is occupied by A atom or +1 if the site is occupied by $\mathrm{B}$ atom. The atomic correlation function $\bar{\Pi}_{k m}(\sigma)$ is the averaged multiplicative of the effective spin variables $\prod S_{i}$ for the cluster $f_{k m}$ in the configuration $\sigma . D_{k m}$ are the degeneracy factor of $f_{k m}$ per site. The effective cluster interaction (ECI) parameter, $\varepsilon_{k m}(V, x)$, denotes the contribution of cluster $f_{k m}$ to the excess energy $\Delta E(\sigma, V, x)$. The ECIs rapidly approach zero as the number of vertices $k$ or the distance between the vertices $m$ in cluster $f_{k m}$ increases for most systems ${ }^{6}$. Therefore, only a limited number of ECIs is needed to describe the excess energy $\Delta E(\sigma, V, x)$ of configuration $\sigma$. In simulations, a set of $N_{J}$ ECIs $\left\{\varepsilon_{k m}\right\}$ can be obtained by mapping the excess energies of $N$ configurations $\{q\}\left(N_{q} \geqslant N_{J}\right)$ from density functional theory (DFT) onto a linear combination of ECIs (see Eq.S1). The convergence and scalability of this set of ECIs $\left\{\varepsilon_{k m}\right\}$ can be verified by comparing 
$\Delta E_{D F T}$ and $\Delta E_{C E}$ through Eq.S1 of other structures which are out of the fitting samples used in the following equation,

$$
\sum_{s}\left|\Delta E_{D F T}(q)-\Delta E_{C E}(q)\right|=\operatorname{Min}
$$

With the well-evaluated ECIs, then, various thermodynamic quantities as a function of temperature, such as configuration stability, phase diagrams, transition temperature $T_{c}$, enthalpy change $\delta H$, can be estimated by using the convergent ECIs $\left\{\varepsilon_{k m}\right\}$ combined with the Monte Carlo (MC) method to solve Eq.S1.

Once the atomic correlation function $\bar{\Pi}_{k m}(\sigma)$ of disordered system at finite temperature are obtained through $\mathrm{MC}$, the averaged electronic structure can be captured by the special quasirandom structure (SQS) method. ${ }^{2,7}$ The SQS can be regarded as an approximation of the average value of the polymorphic configurations. ${ }^{8}$ The electronic band broadening caused by disorder can be obtained by constructing several SQSs and unfolding the band structures of the SQSs with disorder.

\section{Energetics and Short-Range Order Analysis}

In the following parts, the convergent ECIs $\left\{\varepsilon_{k m}\right\}$ are used to investigate the energetics of structures with disorder. The formation energy of a structure with disorder characteristics is defined by referring to the ordered phase that is obtained through many first-principles energy calculations. The formation energy of a disordered structure, usually containing the contributions from enthalpy $\Delta E$ and entropy $\Delta S$, can be decomposed into the configurational (Config) and vibrational (Vib) parts ${ }^{9}$

$$
\Delta F=\Delta F_{\text {config }}+\Delta F_{\text {vib }}=\Delta E_{\text {config }}-T \Delta S_{\text {config }}+\Delta E_{\text {vib }}-T \Delta S_{\text {vib }}
$$

where $\Delta E_{\text {config }}$ is the static formation energy of the MC-based disordered structure with respect to ordered phase from first-principles calculations. By equating the $\mathrm{Nb}$ vacancy disorder to similar case in standard binary alloy, the configurational entropy (per atom) $S_{\text {config }}$ of $\mathrm{Nb}_{0.8} \mathrm{CoSb}$ structures could be estimated by ${ }^{10}$ 


$$
S_{\text {config }}=k_{B} \sum_{i, j=1}^{2}\left[x_{i} p_{i j} \ln \left(\frac{\sum_{k=1}^{2} x_{k} p_{k j}}{x_{i} p_{i j}}\right)\right]
$$

where $x_{i}(\mathrm{i}=1$ or 2$)=0.8$ or 0.2 is the composition of $\mathrm{Nb}(i=1)$ or Vacancy $(i=2)$. $p_{i j}$ represents the chemical environment around atom of $\mathrm{Nb}$ or Vacancy, for example, there are $n p_{11} \mathrm{Nb}$ atoms and $n p_{12}$ Vacancies around a $\mathrm{Nb}$ atom. $n=12$ is the nearest neighbor coordination number. If the Vacancies are randomly distributed on the $\mathrm{Nb}$ sublattice, $p_{i j}=x_{j}$, and $S_{\text {config }}=-k_{B} \sum_{i} x_{i} \ln x_{i}$. So the $S_{\text {config }}$ is bracketed by ${ }^{9}$

$$
0 \leq S_{\text {config }} \leq S_{\text {config }}^{\text {ideal }}=-k_{B} \sum_{i} x_{i} \ln x_{i}
$$

Because of the configuration entropy $S_{\text {config }}$, the structures with disorder characteristics may have lower free energy than ordered structures even at room temperature.

To make comparison with experimental data, electron diffraction pattern is to be simulated for those disorder structures. For a ternary nonstoichiometric compound $\left(\mathrm{Nb}_{\mathrm{x}} \mathrm{Vac}_{1-\mathrm{x}}\right) \mathrm{CoSb}$ which have atom $\mathrm{Nb}$ and Vacancy(Vac) disordering at $\mathrm{Nb}$-sites, the intensity of diffuse scattering caused by the substitutional disorder, $I_{D S R O}$, can be expressed as ${ }^{11,12}$

$$
I_{D S R O}(\mathbf{q})=x(1-x)\left(f_{N b}-f_{V a c}\right)^{2} N \sum_{j=1}^{N} \alpha_{j} \exp \left(i 2 \pi \mathbf{q} \mathbf{R}_{j}\right)
$$

where $\mathbf{q}$ is the diffraction vector $(|\mathbf{q}|=(2 \sin \theta) / \lambda), \quad \mathbf{R}_{j}=r_{n+j}-r_{n}$ denotes the coordinate of a site in crystal lattice relative to the initial site $\mathbf{r}_{\mathrm{n}} \cdot f_{N b}$ and $f_{V a c}$ is the atomic scattering factor of $\mathrm{Nb}$ and Vac. $\alpha_{j}$ is the short-range order parameter. By taking the $\mathrm{Nb}$ sublattice as a regular face-centered cubic (FCC) lattice with lattice constant $a$, the short-range order parameters $\alpha_{h k l}$ at the coordination sphere $(h k l)$ with radius $r=(a / \sqrt{2})\left(h^{2}+k^{2}+l^{2}\right)^{1 / 2}$ is determined by the distribution probabilities of the ' $\mathrm{Vac}-\mathrm{Nb}$ ' pairs,

$$
\alpha_{h k l}=1-\frac{P_{V a c-N b}^{h k l}}{1-x}=1-\frac{1-P_{V a c-V a c}^{h k l}}{1-x}
$$

Here $x=0.2$ is the concentration of Vac. $P_{V a c-N b}^{h k l}\left(P_{V a c-V a c}^{h k l}\right)$ is the probability of finding 
$\mathrm{Nb}$ or Vac on the coordination sphere $(h k l)$ centered at Vac,

$$
P_{V a c-N b(V a c)}^{h k l}=\frac{N_{N b(V a c)}^{h k l}}{N^{h k l}}
$$

where the $N^{h k l}$ is the coordination number of the sphere $(h k l)$, and the $N_{N b(V a c)}^{h k l}$ is the number of $\mathrm{Nb}$ (or $\mathrm{Vac}$ ) on the coordination sphere $(h k l)$. The center Vac should count all the vacancies. Once the short-range order parameters $\alpha_{h k l}$ are determined, the vacancy-vacancy static pair correlation function can be calculated by $P_{V a c-V a c}^{h k l}$.

In principle, the short-range order parameter $\alpha_{h k l}$ characterizes the fluctuations of atomic concentrations in various coordination shells. $\alpha_{h k l}=0$ describes a completely random distribution of vacancies. $\alpha_{h k l}>0$ implies that the Vac-Vac pairs at the specific coordination sphere are more likely to occur in comparison with completely random case, while $\alpha_{h k l}<0$ indicates that the Vac-Vac pairs at the coordination sphere are less likely to occur. The SRO is an atomic distribution state in which a given atomic species are preferentially surrounded by the same or different atomic species in a disordered solid solution, i.e., the occurrence of deviation from the statistical distribution of atoms on the lattice sites. Therefore, the SRO characterizes the radial distribution of atoms, that is, the fluctuation of the atomic concentration in various coordination shells. ${ }^{12}$ In the diffraction pattern, the existence of SRO appears as a three-dimensional diffuse scattering band in reciprocal space ${ }^{11}$.

\section{References}

(1) Morán-López, J. L.; Sanchez, J. M. Theory and Applications of the Cluster Variation and Path Probability Methods; Morán-López, J. L., Sanchez, J. M., Eds.; Springer US: Boston, MA, 1996.

(2) Wei, S. H.; Ferreira, L. G.; Bernard, J. E.; Zunger, A. Electronic Properties of Random Alloys: Special Quasirandom Structures. Phys. Rev. B 1990, 42 (15), 9622-9649. 
(3) Wei, S. H.; Ferreira, L. G.; Zunger, A. First-Principles Calculation of the Order-Disorder Transition in Chalcopyrite Semiconductors. Phys. Rev. B 1992, 45 (5), 2533-2536.

(4) Kikuchi, R. A Theory of Cooperative Phenomena. Phys. Rev. 1951, 81 (6), 988-1003.

(5) Sanchez, J. M.; Ducastelle, F.; Gratias, D. Generalized Cluster Description of Multicomponent Systems. Phys. A Stat. Mech. its Appl. 1984, 128 (1-2), 334-350.

(6) Morán-López, J. L.; Sanchez, J. M. Theory and Applications of the Cluster Variation and Path Probability Methods; Morán-López, J. L., Sanchez, J. M., Eds.; Springer US: Boston, MA, 1996.

(7) Zunger, A.; Wei, S. H.; Ferreira, L. G.; Bernard, J. E. Special Quasirandom Structures. Phys. Rev. Lett. 1990, 65 (3), 353-356.

(8) Trimarchi, G.; Wang, Z.; Zunger, A. Polymorphous Band Structure Model of Gapping in the Antiferromagnetic and Paramagnetic Phases of the Mott Insulators $\mathrm{MnO}, \mathrm{FeO}, \mathrm{CoO}$, and $\mathrm{NiO}$. Phys. Rev. B 2018, 97 (3), 35107.

(9) Van de Walle, A.; Ceder, G. The Effect of Lattice Vibrations on Substitutional Alloy Thermodynamics. Rev. Mod. Phys. 2002, 74 (1), 11-45.

(10) Qin, J. Y. A New Model for the Configurational Entropy of Mixing in Liquid Alloys Based on Short-Range Order. Acta Phys. - Chim. Sin. 2012, 28 (7), 1586-1592.

(11) Welberry, T. R.; Weber, T. One Hundred Years of Diffuse Scattering. Crystallogr. Rev. 2016, $22(1), 2-78$.

(12) Gusev, A. I. Short-Range Order and Diffuse Scattering in Nonstoichiometric Compounds. Physics-Uspekhi 2006, 49 (7), 693-718. 